\title{
Qualitative analysis for state/event fault trees using formal model checking
}

\author{
JIANG Quan ${ }^{1,2}$, ZHU Chunling ${ }^{1, *}$, and WANG Siqi ${ }^{1}$ \\ 1. College of Aerospace Engineering, Nanjing University of Aeronautics and Astronautics, Nanjing 210016, China; \\ 2. China Academy of Electronics and Information Technology, Beijing 100083, China
}

\begin{abstract}
A state/event fault tree (SEFT) is a modeling technique for describing the causal chains of events leading to failure in software-controlled complex systems. Such systems are ubiquitous in all areas of everyday life, and safety and reliability analyses are increasingly required for these systems. SEFTs combine elements from the traditional fault tree with elements from state-based techniques. In the context of the real-time safety-critical systems, SEFTs do not describe the time properties and important timedependent system behaviors that can lead to system failures. Further, SEFTs lack the precise semantics required for formally modeling time behaviors. In this paper, we present a qualitative analysis method for SEFTs based on transformation from SEFT to timed automata (TA), and use the model checker UPPAAL to verify system requirements' properties. The combination of SEFT and TA is an important step towards an integrated design and verification process for real-time safety-critical systems. Finally, we present a case study of a powerboat autopilot system to confirm our method is viable and valid after achieving the verification goal step by step.
\end{abstract}

Keywords: state/event fault tree (SEFT), timed automata (TA), model transformation, safety analysis.

DOI: $10.21629 /$ JSEE.2019.05.13

\section{Introduction}

Software-controlled systems, often called embedded systems, have been widely used in aviation, aerospace, nuclear industry, public transportation, and other safetycritical areas where the failure of these systems will result in heavy property loss, environmental disruption, and even casualties [1]. Fault trees (FTs) or state/event fault trees (SEFTs) are an established methodology for ensuring the safety of software-controlled systems. With the replacement of mechanical and electrical components by software-controlled systems across safety and reliability domains, safety and reliability analysis plays a significant

\footnotetext{
Manuscript received September 10, 2018.

*Corresponding author.

This work was supported by the National Natural Science Foundation of China (11832012).
}

role during the system development [2]. In the field of safety-critical cyber-physical systems (CPSs), where a lot of new interfaces are springing up, Cheng et al. [3] proposed a useful service life prediction method that integrates the stochastic hybrid automata (SHA) model and the dynamic fault tree (DFT) framework to improve the cumulative failure probability (CFP). Song et al. [4] directly mapped multi-state fault trees (MFTs) to Bayesian networks (BNs) to provide a robust probabilistic method for uncertain environments while enabling general diagnostics during analysis and provide the formal definition and reliability analysis for monotone coherent multi-state systems. The traditional DFT analysis methods based on $\mathrm{BN}$ are greatly affected by time segmentation, so Lan et al. [5] proposed an improved dynamic gate transformation method and verified its feasibility and efficiency. In order to do the safety analysis for the distributed component architecture embedded systems, researchers have provided a series of related safety modeling analysis methods [6]. Jin and $\mathrm{Lu}$ [7] focused on the unmanned reconnaissance aerial vehicle (URAV), although no scientific assessment methods have been provided owing to different fight requirements of armed forces, and the authors do the model specification of developing demand, simulate, and calculate some URAV equipment developing scales. The SEFT is a modeling method for expressing the causal chain of a system failure. Roth et al. [8] pointed out that fault tree analysis and Markov chain models have some disadvantages when modeling the hierarchical decomposition, and they improve the method especially with regard to temporal behavior.

FTs are frequently used for the certification of modern safety-critical systems, but FTs have some disadvantages when used to analyze software-intensive systems, especially to model the time-critical software-intensive systems. Roth and Liggesmeyer [9] proposed an approach to obtain the minimal cut set (MCS) through qualitative analysis to identify the most critical components of the sys- 
tem. The top-event indicates the occurrence of failure, and the failure chain is expressed through logic gates and basic components. The SEFT contains the behavior model of the software system, the causality chain of component failure, and the system failure results in the system, so SEFT is suitable for the safety analysis of the componentized embedded software system [10].

Thus, the qualitative analysis of the characteristics of the top-event, which is at the root of the SEFT, is an effective way to obtain system safety properties. However, the SEFT model still lacks a precise semantic description, making it difficult to analyze qualitatively and precisely. Because the SEFT uses both the component features and dynamic behavior semantics of the system, it is difficult to describe and analyze it accurately using methods such as binary decision diagrams. After describing the semantic about the model elements in the SEFT, professionals must still modify the model manually. It is difficult to determine the form of the semantic model of the entire SEFT quickly and efficiently [11].

In this paper, the purpose of our research is to transform the semiformal SEFT modeling language to formal timed automata (TA) language, so we can realize our research goal of providing formal, automated, and qualitative assistance for previous semiformal or informal safety analyses. After the first formal transformation step, we perform model checking for verification of time-related system behavior. There are several model checkers being used in various areas of industrial and academic, such as new symbolic model verifier (NuSMV) [12] and simple Promela INterpreter (SPIN) [13]. However, neither NuSMV nor SPIN supports verification of time-critical system behavior. Then, we use the special real-time model checker UPPAAL to formally validate the accuracy of the SEFT. During model checking, we choose to validate related properties, such as safety (nothing bad should happen), liveness (something good will happen eventually), and deadlock (components sharing the same resource are effectively preventing each other from accessing the resource) [14].

We structure the remainder of this paper as follows. Section 2 presents relevant previous work by our team along with preliminary details of SEFTs, TA, the UPPAAL model checker, and state machine diagrams. In Section 3, we propose a methodology integrating SEFT and TA to perform dynamic and qualitative analyses of the SEFT. Section 4 presents a discussion of the advantages of the methodology and gives a case study to show how the methodology is applied in practice. Finally, we draw conclusions and describe future work in Section 5.

\section{Background and preliminaries}

We have conducted previous research into integrating
SEFTs and TA for qualitative safety analysis. In the previous work, we focused on model transformation, modelbased development, quantitative and qualitative safety analysis, and model verification. SEFTs have been extended to model, analyze, and assess safety-critical systems. The main purpose has been to obtain the minimal cut sequence that leads to system failures. As SEFTs do not provide the precise semantics essential for model verification and minimal cut sequence analysis, $\mathrm{Xu}$ et al. [15] proposed a method to devise a minimal cut sequence for SEFTs based on interface automata. Bozzano and Villaorita [16] proposed an automatic algorithm that devises ordering information and obtains possible constraints based on a formal system model.

Due to the inability of FTs to model the dynamic behavior of complex systems, researchers propose other approaches to model the dynamic behavior and then do the model transformation to verify the safety requirements. In the safety-critical systems domain, increasing numbers of scholars and industry professionals use model-based safety analysis for failure assessment and verification because of the ability to use model transformation and model verification to confirm the existence of failures and combinations of failures in systems that can lead to hazards. Wei et al. [13] proposed a method to analyze a flight control system for potential failures. Ghadhab et al. [17] described the transformation from DFTs into a continuous-time Markov chains (CTMCs) and evaluate various quantitative measures via model checking. Volk et al. [18] presented a new and fast DFT analysis using probabilistic model checking. For the sake of making model-based techniques in industrial software development more efficient, Ding et al. [19] presented a method that combines the respective advantages of finite state machines (FSMs) and model checking technology. Villani et al. [20] combined two formal verification applications - formal UPPAAL and conformance and fault injection (CoFI) - and apply the improved model verification to three cases, finally concluding that the combination of two verification techniques contributes to the identification of a large scale of diversified errors. Wang et al. [21] proposed a method to generate an FT to model system faults and perform the formal model checking, and the authors use a case to demonstrate the effectiveness of the proposed method.

Before introducing our particular method, we need to introduce relevant preliminary material concerning the SEFT, TA, UPPAAL, and state machine diagrams.

\subsection{SEFT}

Owing to the disadvantage of state-based modeling tech- 
nique, compositional modeling, especially causal chains modeling technique, it is becoming too complex and incompetent to fulfill in fault tree analysis (FTA). FTs cannot capture sequences of actions and state history. These shortcomings hamper the application of traditional FTs to software-controlled systems, especially the real-time embedded systems. Thus we use the extended model SEFTs by adding notations of states and events to FTs. States describe conditions that last over a period of time and events describe sudden phenomena and state transitions. Now we give the brief introduction to the SEFT notation elements.

SEFTs are a fault analysis model that combines elements from FTA, state charts, and ROOM charts. We cope with a finite state space for a component that is one active state at each instant of time. SEFTs bridge the gap between traditionally comprehensible FTs and expressive state charts and bring them together into one model. This consolidation makes it possible to model deterministic state spaces and probabilistic failure behavior. Fig. 1 gives the brief overview of the basic symbols in SEFTs [9].

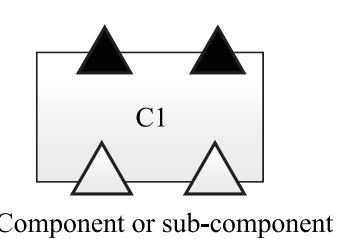

Component or sub-component

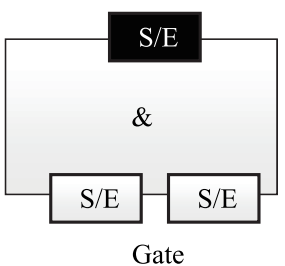

Basic entities

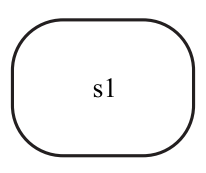

State

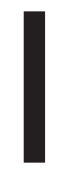

Event

Relations and propositions

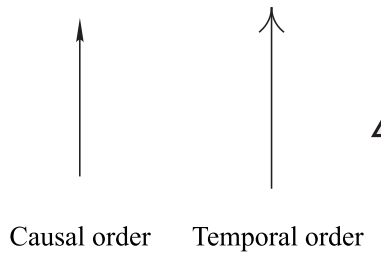

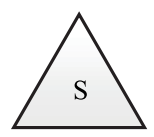

$\mathrm{S}$ S

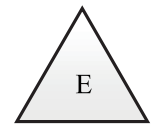

$\mathrm{E}$

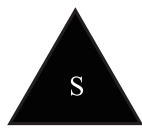

Ports (State input/Event input/ State output/Event output)

Fig. 1 Basic elements of the SEFT component concept

The component concept of SEFT is further developed from component fault trees (CFTs). Each component may be decomposed into subcomponents. Components are selfcontained entities which stand for concurrently technical subsystems in the real world. Components or subcomponents are connected through ports that are typed states ports or event ports in SEFT. This abstraction can be used in both hardware and software systems, and can be applied in the probabilistic behavior modeling in the real word probabilistic environment. Each component is an abstraction of a finite state space, and can be productive for safety and reliability considerations. We denote states by rounded rectangles. Each component is an instantiation of a state at each instant of time. We call a component as the active state and stays in that state for some time interval. Thus the proposition "Component $C$ is in the state $S$ at time $t$ " is either true or false in time $t$. We call any such proposition as a state expression. State expression may be atomic or composed of standard Boolean connectives (AND, OR, NOT...). When considering the probabilistic analysis in SEFT, we can assign a probability in time $t$ for a state expression. States cannot trigger other states or events, meaning that the event can occur only if the state expression evaluates to true.
There are two different kinds of connected edges, firstly in order to model the temporal aspects, we can use the temporal connections with light arrowheads marking the predecessor-successors relation between states and events. Secondly in order to model the enabled relationship between two events, we can use the causal edges with the bold arrowheads marking the triggering relation between two events. Causal edges indicate that each time the source event occurs, the target event occurs as well, provided that it is enabled. Events [2] mentioned just now are denoted by solid bars. Event is the term we use for atomic phenomena that do not spend time to occur. If an event is a transition from one state to another, we call these states predecessor and successor state. In this paper, events occur in one of the three ways:

(i) either they are triggered by other events, or

(ii) they occur after a deterministic delay $t$ or

(iii) after an exponentially distributed probabilistic delay upon entry of predecessor state.

Gate is the junction of causal chains-not restricted to binary or Boolean operators. The most active gates are AND, OR, and NOT in their different variants. SEFT gates have different semantics depending on whether they are 
applied to state terms or to event triggering relations. To model the architecture of a real word system, we can use the component concept provided by SEFTs to communicate with another component and facilitate the failure propagation through in-puts and out-puts. This abstraction enables the modeling of dependencies between events and states among different components. Furthermore, we can refine the in-puts and out-puts into typed ports for states and events indicated by ' $S$ ' or ' $E$ ' in the triangular shape of the respective port. The modeling elements of an SEFT are presented in Fig. 1.

\subsection{TA and timed computational tree logic (TCTL)}

A TA is a state machine extended with clock variables and is a theory for modeling and verification of real-time systems. Generally speaking, we extend a finite automaton with real-valued variables and a finite automaton is a graph containing a finite set of nodes or locations and finite set of labeled edges. The timed automaton can be considered as an abstract model of a real-time system. The clock variables are initialized with zero when the system is started, and then increase synchronously with the same rate. Clock constraints labeled on the edges are used to express the behavior of the automaton. When the clock values satisfy the guard labeled on the edge, a transition represented by an edge can occur. Additionally, TA can communicate with each other through channels, where the sender behavior $x$ ! synchronizes with the receiver behavior $x$ ?

Formally, a TA is defined as a tuple:

$$
\left(N, l_{0}, \text { Lable, } C\right. \text {, clock, guard, invariant) }
$$

where

$N$ is a non-empty, finite set of nodes (locations);

$l_{0}$ is an initial location, $l_{0} \in N$;

$T \subseteq L \times L$ is a set of transitions;

Lable $: N \rightarrow 2^{A P}$ is a function that assigns to each location $l \in N$ with a set $\operatorname{Label}(l)$ of atomic positions;

$C$ is a finite set of clocks;

clock : $T \rightarrow 2^{C}$ is a function that assigns to each transition $t \in T$ a set of clocks $\operatorname{clock}(t)$;

guard : $T \rightarrow \Psi(C)$ is a function that labels each transition $t \in T$ with a clock constraint $\operatorname{guard}(t)$ over $C$;

invariant : $N \rightarrow \Psi(C)$ is a function that assigns to each location (or node) an invariant.

If we want to do verification with the TA model for the real-time systems' behavior, we should model the real-time systems properties specification with a formal language. TCTL is a formal language for property specification based on CTL, and has extended with the clock variables. Usually we can describe expressions in TCTL for state and path formulae in order to do the verification of properties, such as reachability (a system should/can/cannot reach a particular state or states), liveness (something eventually will hold), etc. The syntax supported by UPPAAL for property specification is defined as follows:

$$
\phi::=p|| \phi|\phi \vee \phi| E X \phi|E[\phi \cup \phi]| A[\phi \cup \phi] \mid z . \phi
$$

where $\phi$ is a property to be specified; $p$ is an atomic proposition or a clock constraint; $E X$ is an expression applied on a property; $E$ expresses the existence of a path that fulfills a property; $A$ expresses the invariant fulfillment of a property; $z$ expresses a state predicate.

Next, we introduce the different formulae that can be used in the TCTL syntaxes.

$E[1 p$ There is a path in which the atomic proposition or a clock constraint $p$ will always hold.

$E<>p \quad$ It is possible to reach a state in which $p$ is satisfied.

$A[] p \quad p$ holds invariantly.

$A<>p \quad p$ is inevitable. It will eventually happen.

$A \rightarrow B$ If $A$ becomes true, then $B$ will inevitably be true.

$A$ imply $B$ If $A$ becomes true, $B$ will become true at the same time.

\subsection{UPPAAL model checker}

UPPAAL is a tool for verification of real-time systems jointly developed by Uppsala University and Aalborg University. It has been applied successfully ranging from communication protocols to multimedia applications [22,23]. This tool can be modelled as the networks of TA and be designed to verify the real-time systems. The formal language TA in UPPAAL include integer variables, structured data types, user defined functions, and synchronized channel. The first version of UPPAAL was released in 1995. Since then it has been developed constantly.

In our work, we use UPPAAL as the tool to model TA, simulate the system behavior, and verify the system properties. When we use UPPAAL to model TA, there are two kinds of transitions between states: event-triggering and time-triggering. With regard to event-triggering mechanism, one automaton triggers another automaton via a signal send and receive synchronization through a channel. And for time-triggering mechanism, a transition is fired based on state invariants and time conditions. Next, we briefly exhibit the UPPAAL notation conventions for the real-time system behavior description, which are presented in Table 1. 
Table 1 Notation conventions in UPPAAL for TA figures

\begin{tabular}{|c|c|c|}
\hline Figure & Name & Description \\
\hline \multicolumn{3}{|c|}{ ordinary state } \\
\hline & State & States are represented by circles and (optionally) annotated in red on top of the associated state. \\
\hline & Committed state & $\begin{array}{l}\text { Committed states are represented with circles containing the } c \text { character. Committed states must } \\
\text { be left without time consumption. }\end{array}$ \\
\hline urgent stat & Urgent state & $\begin{array}{l}\text { Urgent states of behaviors are represented with circles containing the } u \text { character. Urgent states } \\
\text { must be left as soon as exiting conditions are found. }\end{array}$ \\
\hline & Initial state & $\begin{array}{l}\text { Initial states are represented by double-lined circles. There must be one unique Initial State per } \\
\text { automaton. }\end{array}$ \\
\hline$t<=$ perio & Invariants & $\begin{array}{l}\text { Invariants should be satisfied in certain states and should be annotated in purple under the related } \\
\text { state. }\end{array}$ \\
\hline & Transition & $\begin{array}{l}\text { Transitions between two states are represented by directional arrows, showing the origin and des- } \\
\text { tination of the transition. }\end{array}$ \\
\hline conditions & Conditions & $\begin{array}{l}\text { Conditions to enable firing of transitions between states are annotated in green under the related } \\
\text { transition. }\end{array}$ \\
\hline signals? & Signals & $\begin{array}{l}\text { Signals used for communication between behaviors are annotated dark blue over the associated } \\
\text { transition. }\end{array}$ \\
\hline & Function & Functions associated with behaviors are annotated in light blue under the associated transition. \\
\hline
\end{tabular}

\subsection{State machine diagram}

The SysML state machine describes the state sequence of a class's objects in response to events during their life cycle and the response generated by the object receiving the event. It is a directed graph of a state node through a transition connection, describing all possible states of a particular object, and the state transitions caused by the occurrence of an event. The basic elements of a state machine typically include states, events, and transitions. State refers to the object in the life cycle including satisfying some conditions, performing some activities, and waiting some events' conditions or situations. The event is the specification of meaningful things occupying a certain position in both space and time, and can be either synchronous or asynchronous. The state transition describes the relationship between the two states of the object, usually composes of five parts: the source state, the triggering event, the guard condition, the transition action, and the target state. In this paper, the software safety qualitative analysis framework based on SEFT and model transformation is presented in Fig. 2.

As shown in Fig. 2, our method first identifies the activities and fault information of the embedded systems using fault and requirement analyses and defines these be- haviors and information as the inputs of the SEFT. From these inputs, we construct the SEFT. We then extend the fault state machine using fault information along with elements semantic mapping and logic gate transformation. We convert the fault state machine to a TA using the model transformation language - atlas transformation language (ATL), including semantic, syntax, and structure transformations. Finally, we specify safety requirements and use the model checking tool UPPAAL to obtain verification results incorporated into the embedded system software design progress.

\section{Research methodology}

Our method transforms the SEFT into a TA. After extending the states, events, interfaces, transitions, and the semantics of logic symbols from the SEFT, we transform this extended state machine into a TA as the inputs verified by the model checker tool UPPAAL.

\subsection{Extension of SEFT to state machine}

The SEFT can be extended to the state machine because the states, events, and internal composition have similar semantics and the tree's logical structure can be described using the state machine representation. We check several 
requirements that must be met for the conversion to a state machine. First, the SEFT must be valid. Second, there must be no nested subcomponents. Third, there must be no input interfaces from external environment to the sub- component. Fourth, there must be no transition relationship between events. Fifth, subcomponents must interact with the containing components. Algorithm 1 shows the implementation algorithm.

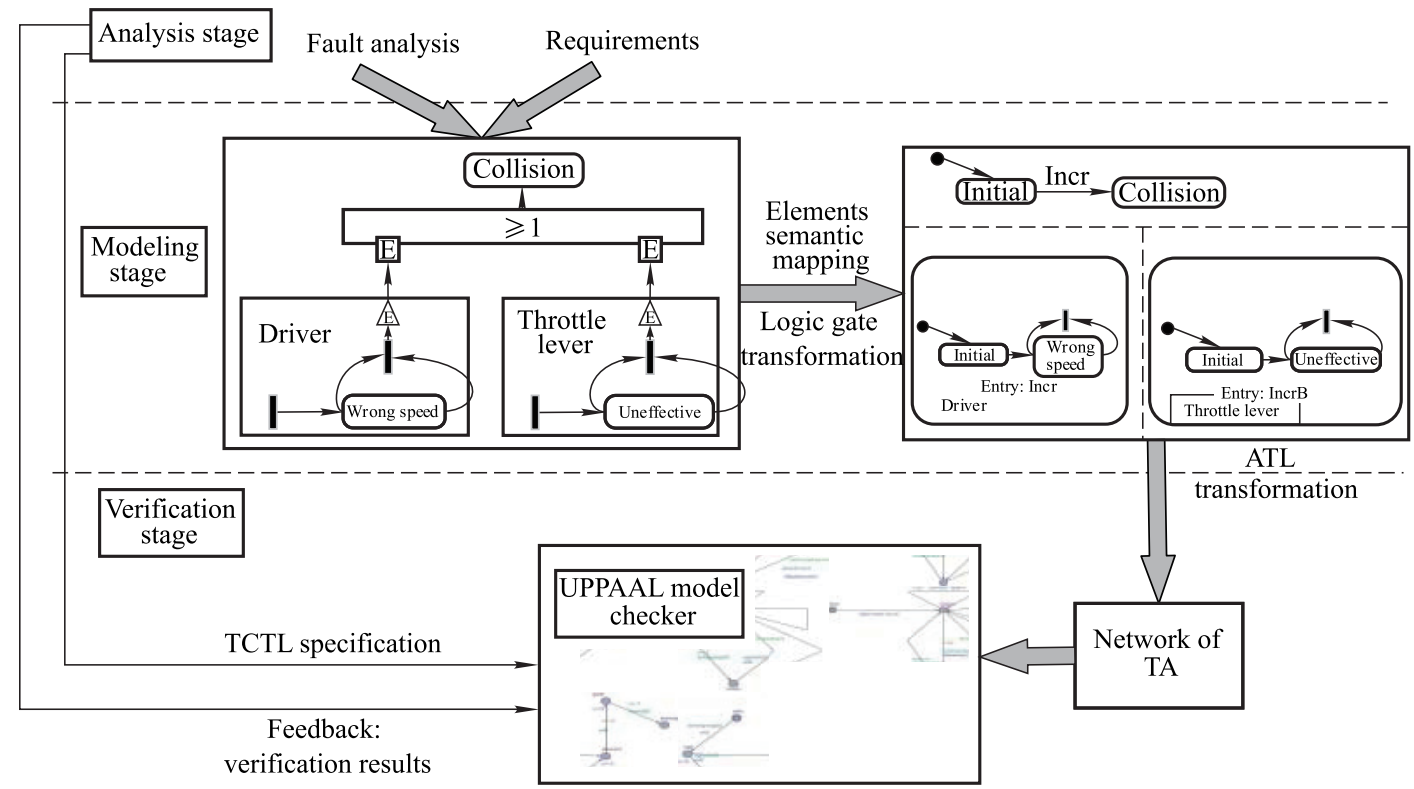

Fig. 2 Software safety qualitative analysis framework based on SEFT and model transformation

Algorithm 1 Judge whether the conditions are met Input: SEFT

Output: component_satisfy, event_satisfy, port_satisfy

Function Name: the judge of transformation condition Initialize (component_satisfy, event_satisfy, port_satisfy)

While (!SEFT.isEmpty())

CurNode=SEFT.node ();

If ( !ComponentEffective(CurNode) ) component_ satisfy=fault; End If;

If $($ CurNode $==$ Event $\& \& N e x t N o d e==$ Event $)$ ) event_ satisfy=fault; End If;

If $($ CurNode $==$ port\& \&PreNode.isEnvironment ()$)$ port_satisfy=fault; End If;

Enqueue(ComponentSet, CurNode)

If (!ExistInteraction(ComponentSet)||ExistNest

(ComponentSet)) component_satisfy=fault; End If;

\section{End While}

Then, the elements including state, event, interface, and transition of the SEFT are mapped to the elements corresponding to the state machine. The core of state mapping is to map the state of the SEFT to the state in the state machine, and the specific implementation algorithm is shown in Algorithm 2.

Algorithm 2 The transformation for states

Input: SEFT

Output: FSM_StateSet[N], FSM_InitState
Function Name: the transformation of state Initialize(FSM_StateSet[N])

While (!SEFT.isEmpty())

CurNode=SEFT.state(); //catching each SEFT state from top to bottom

If(CurNode.isInitState()) FSM_InitState= CurNode; End If; //making the initial state

Enqueue(FSM_StateSet, CurNode)//mapping the current state to the FSM

\section{End While}

Event mapping will map events in the SEFT to events in the state machine, and sort according to the time interval triggered by the events: the first situation is an immediate occurrence; the second situation is an event that is determined either at a predetermined time interval or by an exponential distribution time interval. The implementation algorithm is shown in Algorithm 3.

Algorithm 3 The transformation for events

Input: SEFT

Output: FSM_EventSet[N]

Function Name: the transformation of event

Initialize(FSM_EventSet [N])

While (!SEFT.isEmpty())

CurEvent=SEFT.event();

Enqueue(FSM_EventSet, CurEvent); 


\section{End If;}

If (ExistDelay(CurEvent)) AddInform(FSM_Event);

\section{End While}

The interface in the SEFT is divided into event interface and state interface, and the event interface is mapped to the migration in the state machine. The state interface is mapped to the state in the state machine. The specific implementation algorithm is shown in Algorithm 4.

Algorithm 4 The transformation for ports

\section{Input: SEFT}

Output: FSM_EventSet[N], FSM_TransitionSet[N], FSM_StateSet[N]

Function Name: the transformation of two kinds of port

Initialize(FSM_EventSet[N], FSM_TransitionSet[N], FSM_StateSet[N])

While (!SEFT.isEmpty())

CurNode=SEFT.port();

If(IsEventPort(CurNode)) Enqueue(FSM_EventSet, CurNode); Enqueue(FSM_ TransitionSet, CurNode.)

If(IsStatePort(CurNode)) Enqueue(FSM_StateSet, CurNode);

\section{End While}

The transition of SEFT can be divided into three types: state to state transition, state transition from event to state, and event to event transition. The specific implementation algorithm is shown in Algorithm 5.

Algorithm 5 The transformation for the transition relationships

Input: SEFT

Output: FSM_TransitionSet [N]

Function Name: the transformation of transition relationship

Initialize(FSM_TransitionSet [N])

While (!SEFT.isEmpty())

CurTran=SEFT.transition();

\author{
If(IsState2State(CurTran)) Enqueue(FSM_Transi- \\ tionSet, CurTran.); End If; \\ If(IsEvent2Event(CurTran)) Ignore the current tran- \\ sition; End If \\ If(IsState2Event(CurTran)\&\& IsEvent2State(Next-
} Tran))

$\{$ Enqueue(FSM_TransitionSet, CurTran.); Ignore the next transition; $\}$ End If;

\section{End While}

\subsection{Transformation from logic gate to state machine}

In this section, we will present two kinds of transformational rules from logic AND gates and logic OR gates that are commonly used in SEFT to the corresponding state machine symbols.

\subsubsection{Logic AND gate transformation rules}

The logic AND gate shows that the output event occurs when and only when the input event occurs, and the symbol " $\wedge$ " is used. The AND gate can be represented by the state machine symbol as two orthogonal regions: the input domain and the logical domain. The input field is used to represent the input to the AND gate and contains each of the orthogonal input states. The logical domain is used to represent the logic of the AND gate, representing the interaction between the input events.

As shown in Fig. 3, firstly we assume that the events A and $\mathrm{B}$ are the fault elements in an FT, and belong to the state-occurrence type, so when the component $\mathrm{A}$ reaches the state of $\mathrm{s} 1$ (or component $\mathrm{B}$ reaches state $\mathrm{s} 2$ ), the event Incr will be triggered, component $\mathrm{A} \wedge \mathrm{B}$ will enter state s3 from the initial state. When component A has not exited the s1 state, if component B reaches the s2 state (or component A reaches the s1 state when component B does not exit the s2 state), the Incr event will be triggered again, and component $\mathrm{A} \wedge \mathrm{B}$ will enter the $\mathrm{s} 4$ state.
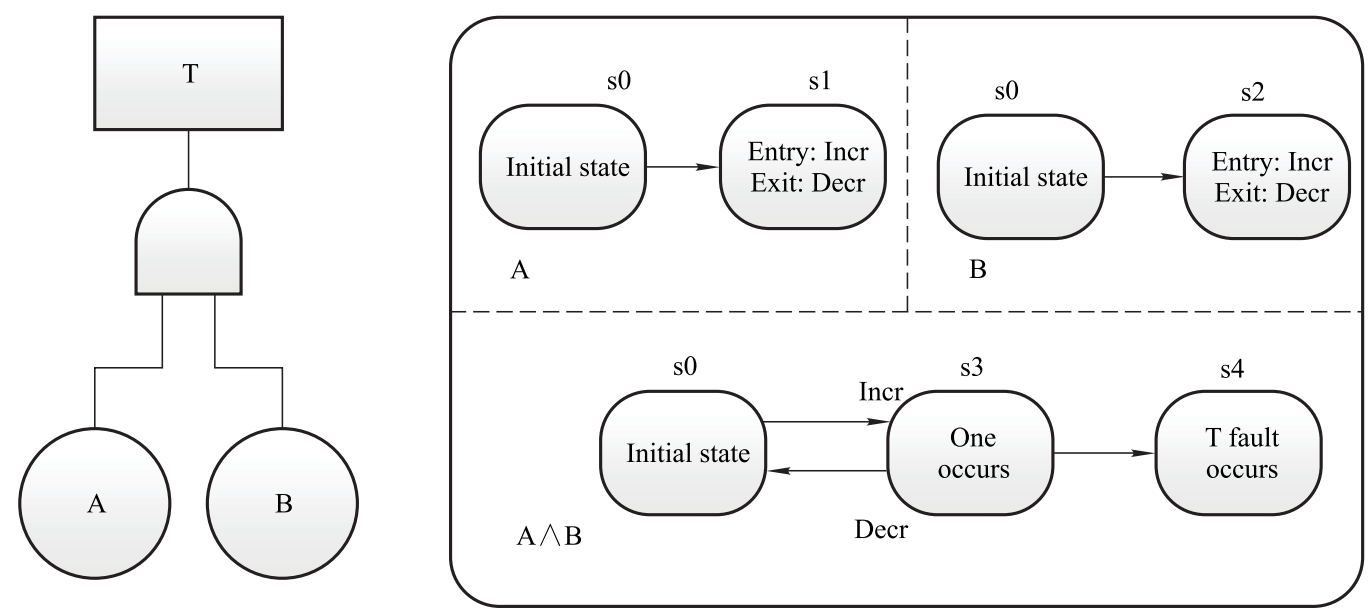

Fig. 3 Logic AND gate and the transformation 
The fault event T occurs. Semantically, when event A and event B occur simultaneously, event $\mathrm{T}$ occurs and is consistent with the fault tree semantics.

\subsubsection{Logic OR gate transformation rules}

The semantics of the logic OR gate is expressed as the output failure event occurs when at least one of the input events occurs, denoted by the symbol " $\vee$ ". Similar to the representation with the logic AND gate, the OR gate can also be represented using two orthogonal state machine regions: input fields and logical domains. When the type of the input event is state arrival, event occurrence, and migration, its input event can be represented in the input field orthogonally. When the input event occurs, the state transition is triggered, and the logic component is transformed from the initial state to the failure state. Fig. 4 depicts an OR gate with two input events, both of which are state arrivals and give the transformed state machine. When component A reaches state $\mathrm{s} 1$ or component B reaches state s2, an incremental event Incr is triggered. After component $\mathrm{A} \vee \mathrm{B}$ receives an incremental event, it transforms from the initial state to output state $\mathrm{s} 3$, and fault event $\mathrm{T}$ occurs. Unlike AND gate logic, in the logical domain of the OR gate, the component $\mathrm{A} \vee \mathrm{B}$ only needs to trigger only one incremental event from the initial state to the failure state, which is consistent with the "at least one event" in the OR gate semantics. When the number of input events increases, it is only necessary to continue to split the input fields and record state transitions for each input event without any changes to the logical domain.
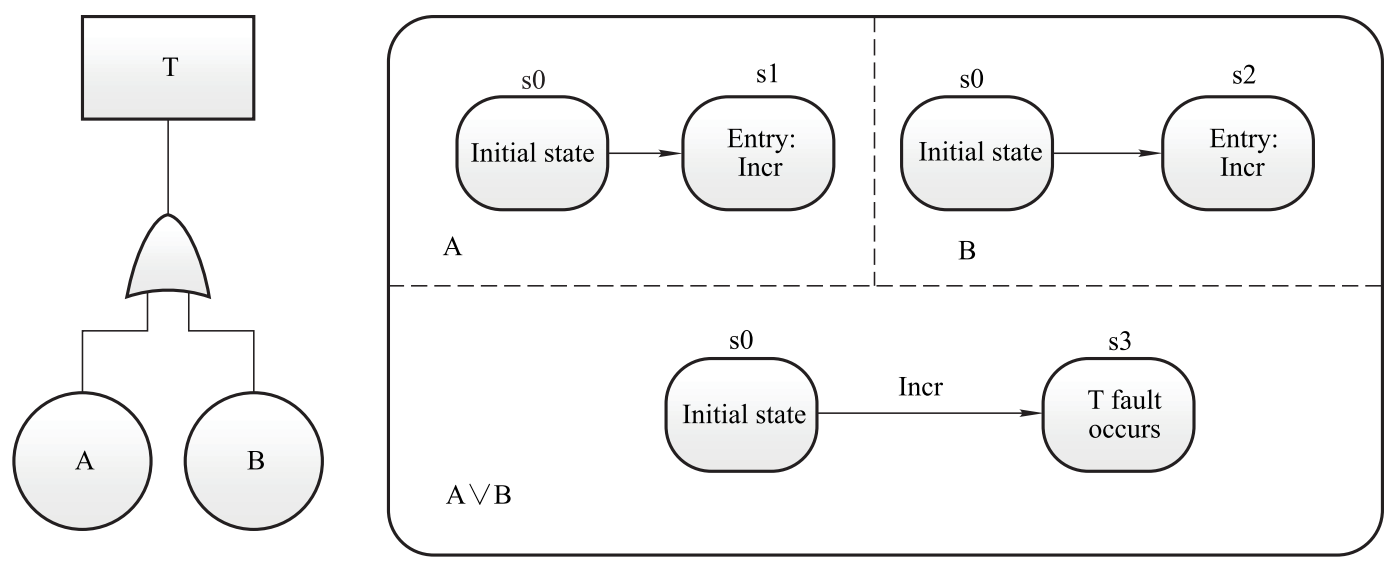

Fig. 4 Logic OR gate and the transformation

\subsection{Transformation from fault state machine to TA}

It is possible to realize the semantic mapping of the fault state machine's data and behavioral elements to the corresponding elements of the TA at the meta-model level. The transformation has certain constraints, some actions will be triggered and some actions will also occur. The state can define invariants and shows constraint conditions. Table 2 shows the mapping from the fault state machine to the meta-model concept of TA.

Table 2 Elements mapping between fault state machine and TA

\begin{tabular}{|c|c|c|}
\hline State machine & TA & Instruction \\
\hline StateMachine & TimedAutomata & Mapping to a TA. \\
\hline Pseudostate & Initial & Expressed as the initial state. \\
\hline State & Location & Indicates the status of the system. \\
\hline Transition & Transition & Corresponds to changes that cause state changes. \\
\hline Constraint & Label & $\begin{array}{l}\text { Constraint can represent state constraints. Because it is an expression in the TA, mapping to Label. } \\
\text { According to whether the object of Constraint is a State or a Transition, the Label also represents the } \\
\text { invariant of Location or the guard of Transition. }\end{array}$ \\
\hline OpaqueBehavior & Label & $\begin{array}{l}\text { The text attribute of the object's body attribute indicates the change caused by the transition, that is, the } \\
\text { expression Exception in Label. }\end{array}$ \\
\hline Trigger & Action & $\begin{array}{l}\text { The trigger triggered by the trigger maps to Action, and the Trigger maps to Synchronisation, which } \\
\text { indicates the operation of the transition synchronization. This allows us to define synchronization events } \\
\text { for different TA. }\end{array}$ \\
\hline
\end{tabular}


There is a concurrency structure in the state machine; in UPPAAL, the processing of the concurrency structure is not expressed using a TA that has a concurrency structure, but a concurrent combination of TA that does not have multiple concurrency structures. Therefore, the work in this paper needs to transform the state machine with a single existing concurrency structure to multiple TA with concurrent combinations.

Transformation from fault state machine of the concurrent structure to TA network as Algorithm 6.

Algorithm 6 The transformation of concurrent structure

Input: $\mathrm{SMD}=\left(S^{\mathrm{in}}, S^{\text {out }}\right.$, guard, transition, trigger, lable);

Output: TA $=($ disabled, firing, clock, go, edge, guard, lable);

Function: transform SMD into NTA

\section{Begin}

declare TA: go, one edge, one place

for (every state $S^{\text {in }}$ )

$\{$ declare variable $s$ in NTA

if ( isthisState( ) ) $s=1$;

else $s=0$;

\}

if ( isTransition( ) )

add disabled, firing, Clock c;

add go? on edge from disabled to firing;

if ( transtitionHasTrigger( ) )

add trigger on edge; trigger is the synchronization;

else go is the synchronization;

end if;

if ( sHaslable( ) ) add lable to firing;

end if;

add edge from disabled to firing;

add guard on edge;

update $s_{1}^{\text {in }}==l \& \& \ldots \& \& s_{n}^{\text {in }}==1 \& \& c==0$;

add edge from firing to disabled;

add guard on edge;

update $s_{1}^{\text {in }}==1 \& \& \ldots \& \& s_{1}^{\text {in }}==1$;

if ( transtitionHasTrigger( ) )

update $s_{1}^{\text {in }}==0 \ldots s_{1}^{\text {in }}==0, s_{1}^{\text {out }}==$

$1 \ldots s_{1}^{\text {out }}==1$

\section{end if;}

add edge from firing to disabled;

guard.lable = lable;

update $s_{1}^{\text {in }}==0 \ldots s_{1}^{\text {in }}==0, s_{1}^{\text {out }}==$

$1 \ldots s_{1}^{\text {out }}==1$

end if;

end

\section{Case study}

As a test case, we analyze the example of a powerboat autopilot (PBA), build a speed control FT for collisions, and expand it into a SEFT for the PBA system. Using the ATL transformation platform, we transform the SEFT into a TA and then input the TA into UPPAAL to verify the speed properties.

\subsection{System overview}

The PBA includes sensors, actuators, interface and display units, and the computer hardware needs to provide powerboat navigation and control. The control device is a key component of the PBA. It calculates and analyzes the current speed from the speed sensor and the position information from the GPS unit, provides appropriate commands for speed control (such as throttle), and controls the powerboat at an appropriate cruising speed. At the same time, the PBA receives speed input from the driver and provides the driver with a data display. The driver provides the PBA control system with the desired speed and standard operating parameters (e.g., future locations, waypoints, and desired speeds). One of the multiple waypoints is calibrated as the final waypoint. When the final waypoint is reached, the PBA gradually reduces the motor speed to idling and shows the user arrival. At other times, the display shows the system status including current speed, desired speed, current position, desired position (next waypoint), and system status (initialization, idle, control, shutdown). In addition, the driver can activate the braking device to terminate all control actions of the PBA and force the powerboat to slow to a stop.

In formulating the requirements of the PBA control device, we assume that there are six self-driving powerboats and three docks. In order to avoid the collision, only one powerboat may be in the berthing process at a time. The requirements are as follows.

(i) The system should provide the driver with a real-time display of the current speed and position. When a selfdriving powerboat approaches the docking point (the nearest sailing point), it should send a berthing request signal. If the dock does not have a free berthing point, it will receive the reply that it is not allowed to berth. The reply should be received within two moments. If there is a free dock, a berthing approval reply will be received.

(ii) The system will monitor the driver's input information. In the case of an emergency, the driver may activate the emergency braking device, signaling the system to reduce the powerboat's speed to 0 . Starting the emergency braking device early will cause the powerboat to fail to 
reach waypoints. These failures are marked as faults.

(iii) The speed control module or the driver's input gives velocity commands so that the powerboat sails to the waypoint at the appropriate speed (not exceeding the critical speed) and drops the speed to 0 when it reaches the desired position.

(iv) After receiving a "docking allowed" reply, the system must gradually reduce the motor speed to idle within two to three moments and display "docking" to the user. The powerboat is then driven to the dock and the speed is reduced to 0 through inertia within one to two moments. When docking is not permitted, the powerboat needs to drift on the water. Once the dock is idle, the powerboat system receives the signal to allow the powerboat to berth.

\subsection{FT constructing of collision accident}

According to these autopilot safety requirements, we ana- lyze the following collision event: If a speed control operation fails or the speed control device fails, the powerboat could not reduce the speed to 0 at the predetermined location, resulting in a collision. The speed control operation failure could be due to inputting the wrong desired speed (e.g., too fast). The speed control equipment failure could be due to a failure of the speed alert device (e.g., speed sensor or display failure) or a failure of the throttle (throttle caused by the empty joystick caused by emptying the throttle). From these circumstances and possible causes, we construct a fault tree in which a collision accident occurs when the launcher reaches the waypoint, as shown in Fig. 5. According to Fig. 5, we can construct the corresponding SEFT for the collision accident as shown in Fig. 6. Using the research methodology described in Section 3, we obtain the associated state machine diagram in Fig. 7.

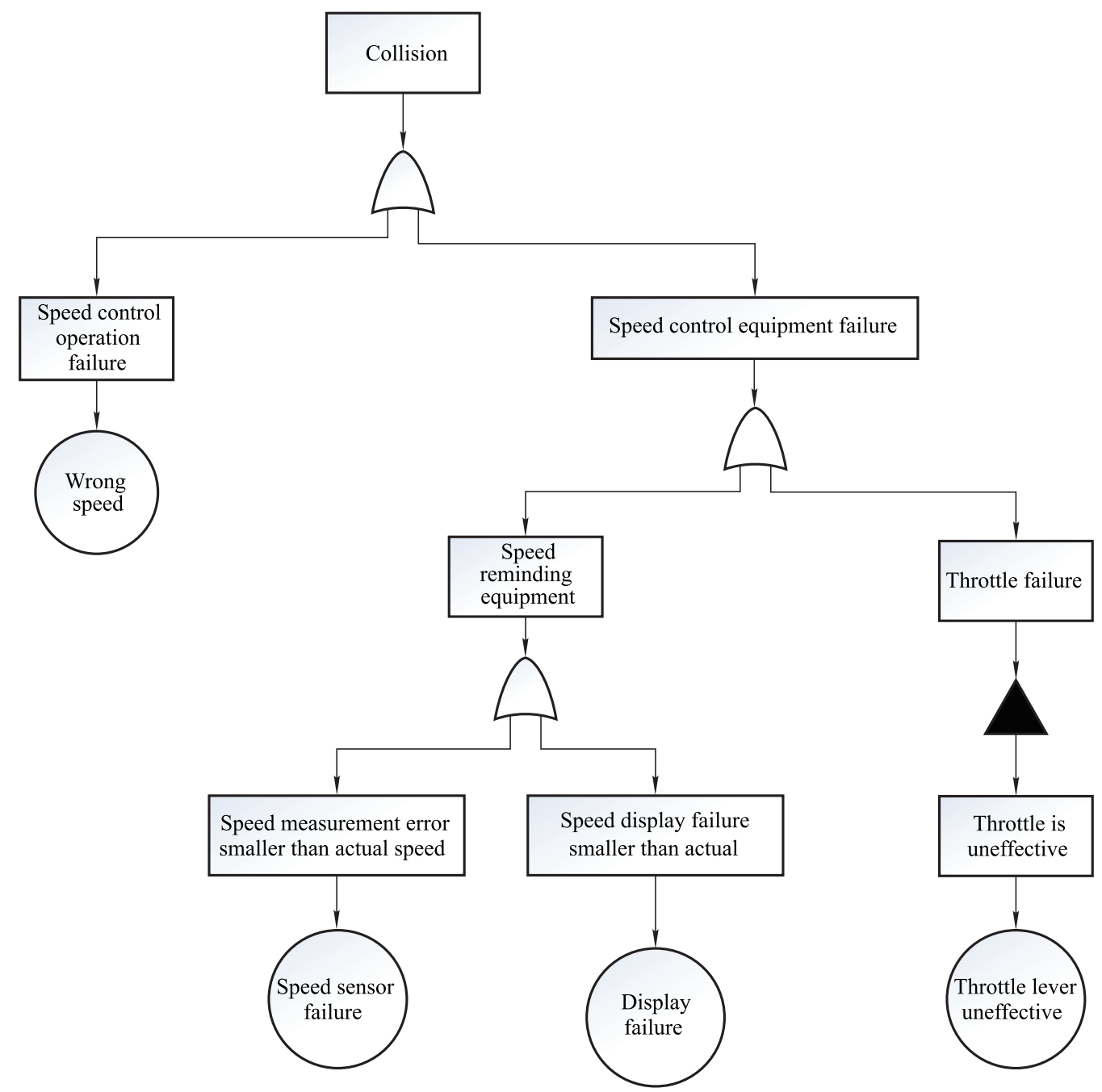

Fig. 5 FT in collision of PBA system 


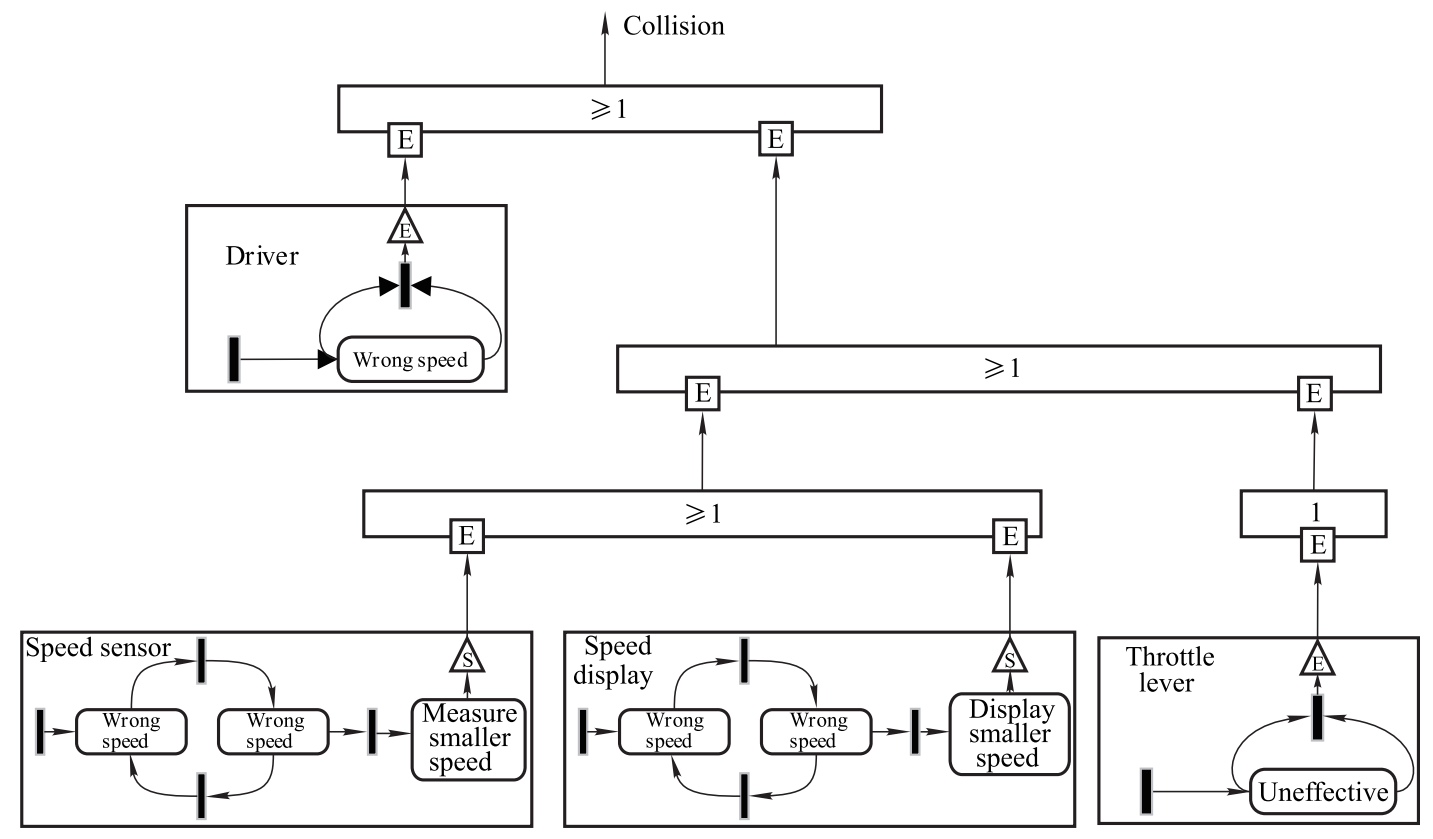

Fig. 6 SEFT in collision of PBA system

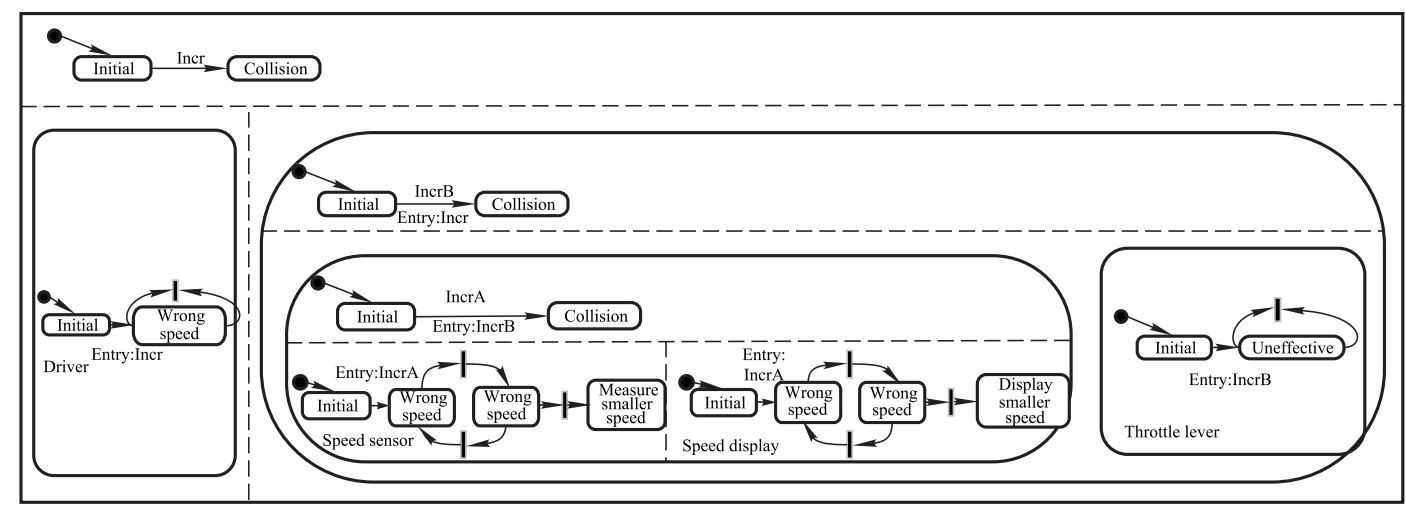

Fig. 7 State machine of PBA

\subsection{System simulation and safety properties verification}

(i) Real-time simulation for time automaton network model

According to the PBA system requirements described in Section 4.1, firstly, we could construct the time automaton network model of the powerboat docking system, which is composed of multiple PBA TA model in Fig. 8(a) and control TA model in Fig. 8(b). UPPAAL simulates these above TA. Fig. 9 shows a fragment of the message control sequence and describes the synchronization and state transitions of messages between multiple PBA and control models in the TA network. Through synchronous messages such as stopReq, agree, notagree and leave, self-driving powerboats carry out the safe docking process. After simu- lation and validation, it is found that the model definition, variable definition, and the syntax are all correct and that the system function and safety requirements are met. Thus, the model construction is correct.

(ii) Safety properties verification for time automaton network model

In this section, we also use UPPAAL to do further verification and analysis. First, verify whether there is a deadlock in the system and enter the $A[]$ !deadlock property in the verifier. Deadlock detection is directly supported by the model checker UPPAAL, as mentioned before, the property deadlock means that components sharing the same resource are effectively preventing each other from accessing the resource. The verification result shows that the deadlock property is satisfied, and there is no deadlock in the system. 


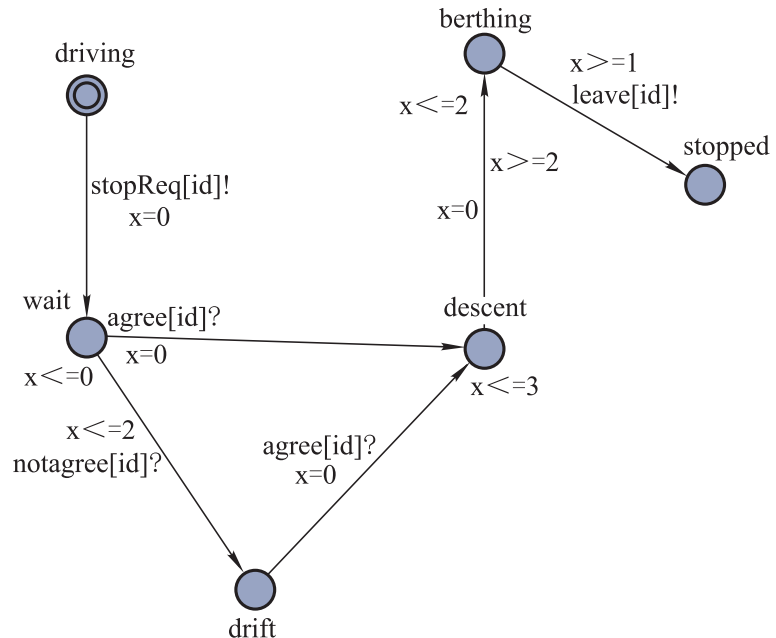

(a) Automaton of PBA

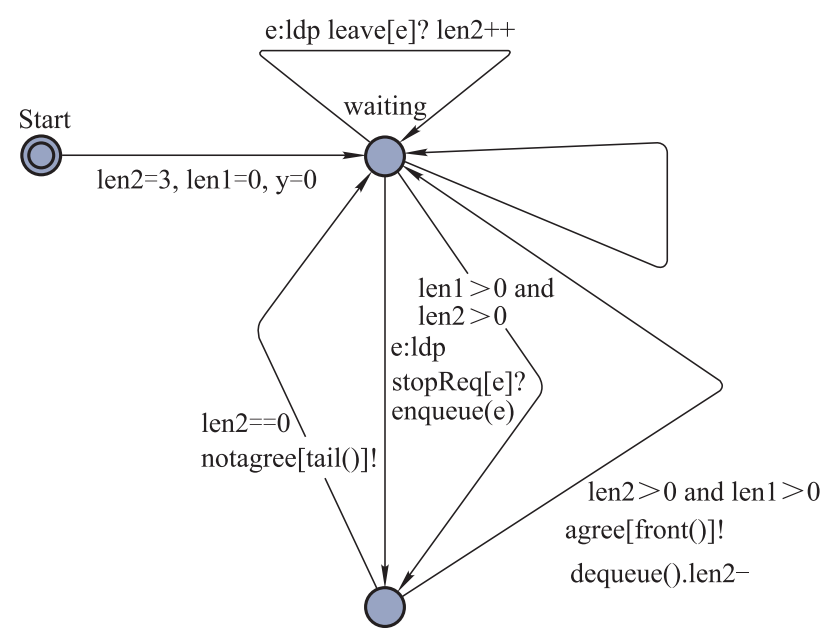

(b) Automaton of control

Fig. 8 TA of PBA and control

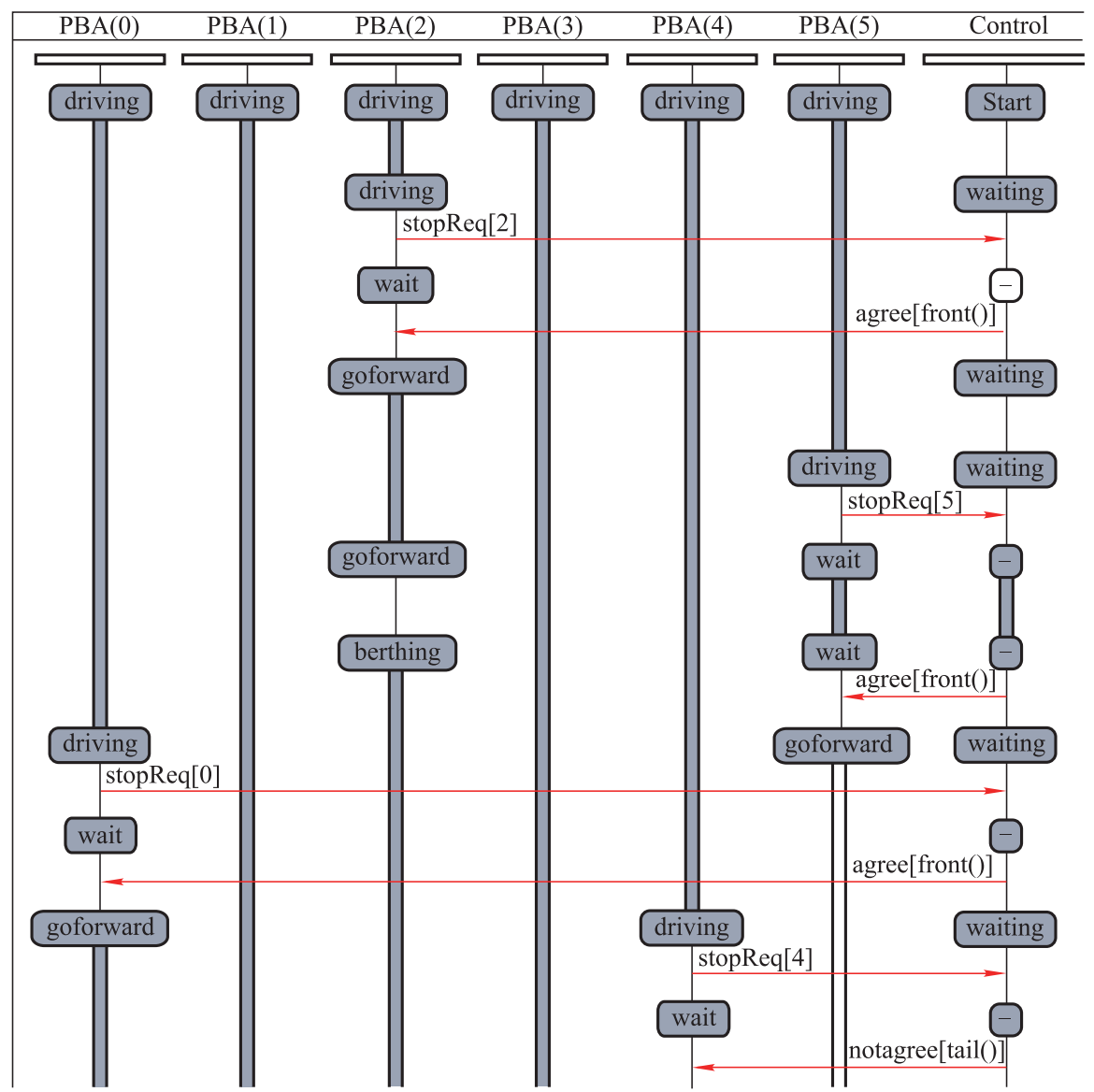

Fig. 9 Real-time simulation for TA network model

Next, we verify the system safety according to the safety properties specification obtained in Section 4.2, the property of safety means that nothing bad should happen due to the errors within the system or software, and behavioral safety [14] refers to a desired behavior of a system that would not crash or change to undesired behavior during runtime when encountering errors. The following assumptions are made: when the device fails (for example, the sensor cannot work, the display is abnormal, the throttle lever fails, etc.), non-human participation in repair equip- 
ment cannot return to normal on the device own. Therefore, the fault event generated at any time before the fault occurs may cause the final mishap. The safety property to be verified play a warning role while we want to verify the whole system safety. For the speed control module subtree, driver input errors, sensor failures, and display failures could all cause speed control failures that should be verified as the safety properties. This can be equivalent to the occurrence of a mishap event as the resulting failure. Finally, we should verify the liveness property which means that something good will happen eventually, and the reachability property which means that there is a finite path for reaching a state. The state is reachable as a verification input and is verified in the UPPAAL model checker. We then demonstrate the representative deadlock, safety, liveness and reachability properties obtained from the SEFT analysis.

First, there is no deadlock in the system, and we can verify this property specification using TCTL in the formula:

(i) A[]!deadlock!;

Then, according to the previous paragraph, we describe the safety property using these TCTL formulae:

(ii) $A[]$ Control.list $[P-1]==0$

It Indicates that the docking list of the controller will not have an overflow status.

(iii) $A[] P B A(0)$.berthing \&\&PBA(1).berthing imply Control.len $2<=1$

It indicates that two self-driving powerboats will not be docked at the same berth at the same time which will lead to collision or mishap.

Finally, according to the functional requirements of the self-driving powerboat docking scenario, the time automata network must satisfy the following verification properties to ensure the correctness of the system logic and the liveness of time constraints. And we can do this property specification using TCTL in this formula:

(iv) $E \ll P B A(0)$.stopped and $P B A(1)$.stopped and $P B A(2) . s t o p p e d$ and $P B A(3)$. stopped and PBA(4).stopped and PBA(5).stopped and Control.waiting

It shows that each self-driving powerboat can be safely and orderly docked at the port.

Fig. 10 shows the verification result. The green circle indicates that the deadlock, safety, liveness, and reachability properties are satisfied, that the normal and continuous functionality of the berthing process are ensured, and that the docking requirements of each PBA are met safely and eventually.

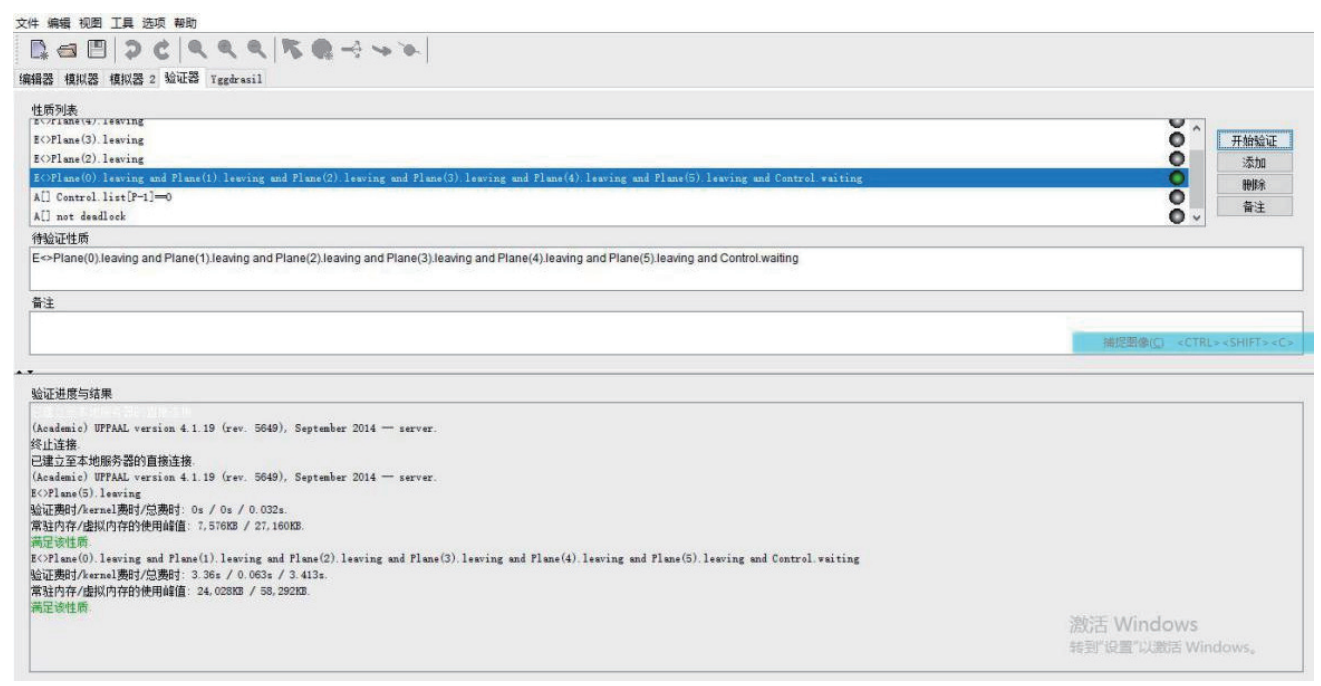

Fig. 10 Formal verification for PBA in the time model checker UPPAAL

In summary, the berthing time will take less than three moments and the collision will not occur. According to the verification results, we can recheck the process of system design, especially with regard to the safety-critical time property. The system lacks functions for obtaining the desired speed command and speed information transmitted by the sensor. Corrections should be made to the above problem in the system when the driver gives the desired speed command, current position, and target dock position, etc. The critical speed must be calculated based on the above information to determine whether the input speed is appropriate, and to give a warning when the critical speed is exceeded. Similarly, for the speed value obtained by the sensor and the speed information displayed on the view- 
ing screen, related confirmation functions need to be added to ensure the safe operation of the powerboat during autonomous driving, with an emergency braking reminder provided in the event of an emergency.

\section{Conclusions}

In this paper, we use the SEFT to model embedded safetycritical hardware and software and integrate the functional model and safety requirements. Through states, events, interfaces, migrations, and other extensions, a state machine diagram can be skillfully modelled by software engineering personnel. Finally, it is transformed to the TA. On the one hand, this method adds a description of fault logic on the basis of preserving the functional description of the state machine diagram for the system, so that it can simultaneously describe the system's safety requirements and functional behavior, and is more suitable for safety analysis. Therefore, the model can quickly find out the process of the fault and the software behavior that causes the fault, so as to facilitate the modification of the behavior model and satisfy the safety requirements of the system function model.

In this paper, we focus on qualitative analysis for SEFT using formal transformation and model checking. In the future work, we will focus on quantitative analysis for SEFT, especially in the dynamic environment, using the stochastic model checking technology, we can do the dynamic reliability analysis for the accident prediction and elimination which is very important for safety-critical systems.

\section{Acknowledgment}

The authors would like to thank the editors and all anonymous referees whose comments and suggestions greatly helped us to improve the presentation and the quality of this paper. Also, we would like to thank the humancomputer environmental engineering laboratory that the authors belong to, for the great help given by the team in the experiment analysis of this paper.

\section{References}

[1] DASKAYA I, HUHN M, MILIUS S. Formal safety analysis in industrial practice. Proc. of the International Conference on Formal Methods for Industrial Critical Systems, 2011: 68 - 84 .

[2] KAISER B, GRAMLICH C. State-event-fault-trees — a safety analysis model for software controlled systems. Reliability Engineering \& System Safety, 2007, 92(11): 1521 - 1537.

[3] CHENG Y H, JIANG L, JIANG B, et al. Useful life prediction using a stochastic hybrid automata model for an ACS multigyro subsystem. Journal of Systems Engineering and Electronics, 2019, 30(1): 154-166.

[4] SONG B, ZHOU Z, MA C, et al. Reliability analysis of monotone coherent multi-state systems based on Bayesian networks. Journal of Systems Engineering and Electronics, 2016, 27(6):

$$
1326-1335 \text {. }
$$

[5] LAN J, YUAN H J, XIA J. Improved method for dynamic fault tree analysis based on discrete-time Bayesian network. Systems Engineering and Electronics, 2018, 40(4): 948 -953. (in Chinese)

[6] ČEPIN M, MAVKO B. A dynamic fault tree. Reliability Engineering \& System Safety, 2002, 75(1): 83-91.

[7] JIN G D, LU L B. System of developing scale modeling and simulation for URAV. Journal of Systems Engineering and Electronics, 2008, 19(6): 1295 - 1300.

[8] ROTH M, HARTOYO A, LIGGESMEYER P. Efficient reachability graph development for qualitive analysis of state/event fault trees. Proc. of the IEEE International Symposium on Software Reliability Engineering Workshops, 2015: 144-151.

[9] ROTH M, LIGGESMEYER P. Qualitative analysis of state/event fault trees for supporting the certification process of software-intensive systems. Proc. of the IEEE International Symposium on Software Reliability Engineering Workshops, 2013: $353-358$.

[10] KABIR S. An overview of fault tree analysis and its application in model based dependability analysis. Expert Systems with Applications, 2017, 77: 114-135.

[11] BOUDALI H, CROUZEN P, STOELINGA M. Dynamic fault tree analysis using input/output interactive Markov chains. Proc. of the IEEE/IFIP International Conference on Dependable Systems and Networks, 2007: 708-717.

[12] DAL LAGO L, FERRANTE O, PASSERONE R, et al. Dependability assessment of SOA-based CPS with contracts and model-based fault injection. IEEE Trans. on Industrial Informatics, 2018, 14(1): 360-369.

[13] WEI Q, JIAO J, ZHAO T. Flight control system failure modeling and verification based on SPIN. Engineering Failure Analysis, 2017, 82: $501-513$.

[14] IGLESIA D G D L, WEYNS D. MAPE-K formal templates to rigorously design behaviors for self-adaptive systems. ACM Trans. on Autonomous and Adaptive Systems, 2015, 10(3): $1-30$.

[15] XU B, HUANG Z, HU J, et al. Minimal cut sequence generation for state/event fault trees. Proc. of the ACM Middleware Doctoral Symposium, 2013, DOI: 10.1145/2541534.2541592.

[16] FEILER P, DELANGE J. Automated fault tree analysis from AADL models. ACM SIGAda Ada Letters, 2017, 36(2): $39-$ 46.

[17] GHADHAB M, JUNGES S, KATOEN J P, et al. Safety analysis for vehicle guidance systems with dynamic fault trees. Reliability Engineering \& System Safety, 2019, 186: 37 - 50.

[18] VOLK M, JUNGES S, KATOEN J P. Fast dynamic fault tree analysis by model checking techniques. IEEE Trans. on Industrial Informatics, 2018, 14(1): 370-379.

[19] DING Y, LI W, ZHONG D, et al. System states transition safety analysis method based on FSM and NuSMV. Proc. of the 2nd International Conference on Management Engineering, Software Engineering and Service Sciences, 2018: $107-$ 112.

[20] VILLANI E, PONTES R P, CORACINI G K, et al. Integrating model checking and model based testing for industrial software development. Computers in Industry, 2019, 104: $88-$ 102.

[21] WANG L S, LI S J, WEI O H, et al. An automated fault tree generation approach with fault configuration based on model checking. IEEE Access, 2018, 6: 46900-46914.

[22] GERD B, ALEXANDRE D, KIM G. A tutorial on UPPAAL 
4.0. Department of Computer Science, 2006, 4(12): 200-236.

[23] IGLESIA D G D L, WEYNS D. MAPE-K formal templates to rigorously design behaviors for self-adaptive systems. ACM Trans. on Autonomous and Adaptive Systems, 2015, 10(3): $1-31$.

\section{Biographies}

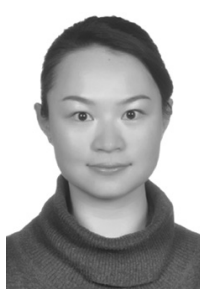

JIANG Quan was born in 1981. She is a doctor candidate in Nanjing University of Aeronautics and Astronautics, majoring in man-machine and environmental engineering. Her main research interests are embedded system fault analysis, system safety analysis, and formal verification and validation.

E-mail: jiangquan@nuaa.edu.cn

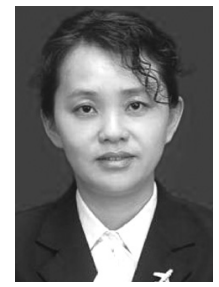

ZHU Chunling was born in 1968. She is a professor in Nanjing University of Aeronautics and Astronautics, and her research lies in the fields of aircraft environment control, aircraft icing and protection, airborne equipment cooling, with a particular focus on man-machine and environmental engineering. E-mail: clzh@nuaa.edu.cn

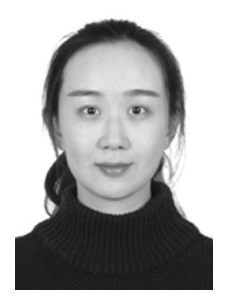

WANG Siqi was born in 1990. She graduated from Nanjing University of Aeronautics and Astronautics in 2016, majoring in software engineering. And now she works in Huawei Company, mainly responsible for the safety design and analysis of electronic products. Her research interests include system modeling, requirement verification, and formal model checking.

E-mail: wangsq@nuaa.edu.cn 